\title{
Interactions of Carbohydrate Intake and Physical Activity with Regulatory Genes Affecting Glycaemia: A Food4Me Study Analysis
}

\author{
Santiago Navas-Carretero a, b, c Rodrigo San-Cristobal ${ }^{\mathrm{a}} \quad$ Ismael Alvarez-Alvarez $^{\mathrm{a}}$ \\ Carlos Celis-Morales $^{d, e}$ Katherine M. Livingstone ${ }^{d, f}$ Claire B. O'Donovan $^{g}$ \\ Christina Mavrogianni $^{\text {h }}$ Christina P. Lambrinou ${ }^{\text {h }}$ Yannis Manios $^{\text {h }}$ Iwona Traczyck ${ }^{\mathrm{i}}$ \\ Christian A. Drevon $^{j}$ Cyril F.M. Marsaux ${ }^{k}$ Wim H.M. Saris ${ }^{k}$ Rosalind Fallaize ${ }^{l, m}$ \\ Anna L. Macready ${ }^{m}$ Julie A. Lovegrove ${ }^{m}$ Thomas E. Gundersen ${ }^{n}$ Marianne Walsh ${ }^{9}$ \\ Lorraine Brennan $^{g}$ Eileen R. Gibney ${ }^{g}$ Mike Gibney ${ }^{g}$ John C. Mathers ${ }^{d}$ \\ J. Alfredo Martinez $z^{a, b, c}$ on behalf of Food4Me \\ ${ }^{a}$ Centre for Nutrition Research, School of Pharmacy and Nutrition, University of Navarra, Pamplona, Spain; \\ ${ }^{b}$ CIBEROBN, Instituto de Salud Carlos III, Madrid, Spain; ' IdiSNA, Navarra Institute for Health Research, Pamplona, \\ Spain; ${ }^{d}$ Human Nutrition Research Centre, Population Health Sciences Institute, Newcastle University, Newcastle

 \\ University of Glasgow, Glasgow, UK; f Institute for Physical Activity and Nutrition, School of Exercise and Nutrition \\ Sciences, Deakin University, Geelong, VIC, Australia; 9UCD Institute of Food and Health, University College Dublin,

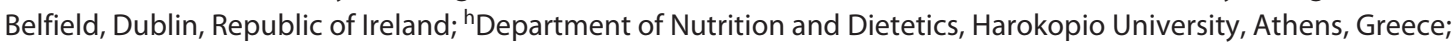 \\ 'Department of Human Nutrition, Faculty of Health Sciences, Medical University of Warsaw, Warsaw, Poland; \\ jDepartment of Nutrition, Institute of Basic Medical Sciences, Faculty of Medicine, University of Oslo, Oslo, Norway; \\ kDepartment of Human Biology, NUTRIM, School for Nutrition and Translational Research in Metabolism, Maastricht \\ University Medical Centre, Maastricht, The Netherlands; 'School of Life and Medical Sciences, University of \\ Hertfordshire, Hatfield, UK; ${ }^{\mathrm{m} H u g h}$ Sinclair Unit of Human Nutrition and Institute for Cardiovascular and Metabolic \\ Research, University of Reading, Reading, UK; ${ }^{n}$ Vitas Analytical Services AS, Oslo Science Park, Oslo, Norway
}

\section{Keywords}

Carbohydrate intake - Type 2 diabetes · Glucose

metabolism · Physical activity · Genetics · Ponderal status

\begin{abstract}
Introduction: Carbohydrate intake and physical activity are related to glucose homeostasis, both being influenced by individual genetic makeup. However, the interactions between these 2 factors, as affected by genetics, on glycaemia have been scarcely reported. Objective: We focused on analysing the interplay between carbohydrate intake and phys-
\end{abstract}

ical activity levels on blood glucose, taking into account a genetic risk score (GRS), based on SNPs related to glucose/ energy metabolism. Methods: A total of 1,271 individuals from the Food4Me cohort, who completed the nutritional intervention, were evaluated at baseline. We collected dietary information by using an online-validated food frequency questionnaire, a questionnaire on physical activity, blood biochemistry by analysis of dried blood spots, and by

Current address of Rodrigo San-Cristobal and J. Alfredo Martinez: IMDEA-Food, Research Institute on Food and Health Sciences, Program of Precision Nutrition, CSIC-UAM, Madrid, Spain.
C 2021 The Author(s).

Published by S. Karger AG, Basel

This article is licensed under the Creative Commons AttributionNonCommercial-NoDerivatives 4.0 International License (CC BY NC-ND) (http://www.karger.com/Services/OpenAccessLicense) Usage and distribution for commercial purposes as well as any distribution of modified material requires written permission.
Correspondence to:

Santiago Navas-Carretero, snavas@unav.es 
analysis of selected SNPs. Fifteen out of 31 SNPs, with recognized participation in carbohydrate/energy metabolism, were included in the component analyses. The GRS included risk alleles involved in the control of glycaemia or energyyielding processes. Results: Data concerning anthropometric, clinical, metabolic, dietary intake, physical activity, and genetics related to blood glucose levels showed expected trends in European individuals of comparable sex and age, being categorized by lifestyle, BMI, and energy/carbohydrate intakes, in this Food4Me population. Blood glucose was inversely associated with physical activity level $(\beta=$ $-0.041, p=0.013$ ) and positively correlated with the GRS values ( $\beta=0.015, p=0.047)$. Interestingly, an interaction affecting glycaemia, concerning physical activity level with carbohydrate intake, was found ( $\beta=-0.060, p=0.033$ ), which also significantly depended on the genetic background (GRS). Conclusions: The relationships of carbohydrate intake and physical activity are important in understanding glucose homeostasis, where a role for the genetic background should be ascribed.

(c) 2021 The Author(s).

Published by S. Karger AG, Basel

\section{Introduction}

Metabolic pathways affecting body composition and metabolic markers involve complex interactions concerning energy metabolism [1] including lipid and glucose transport [2], which are under genetic control. Thus, the consumption of different proportions of carbohydrates and lipids, together with the type, modality, and duration of exercise, has an impact on body physiology and composition $[3,4]$. In addition, individual genetic makeup may also influence energy utilization and macronutrient metabolism $[5,6]$. Indeed, the dietary content of protein, carbohydrates, or fibre, as well as the amount of fat, is important for plasma glucose concentration [69]. Moreover, a potentially causal link between fibre intake and diabetes incidence has been reported [10]. Genotype may also affect how different macronutrients influence glycaemia [11], mediated by the expression of genes related to insulin function, glucose utilization, and transport or energy homeostasis as well as macronutrient metabolism [12].

Increased physical activity is a useful strategy to reduce body weight [13] and related complications, such as high glucose levels, whose outcomes depend not only on the exercise type and duration but also on the individual's genotype $[14,15]$. Some studies have assessed interactions between physical activity and macronutrient distri- bution on management of chronic diseases [16-18], but the results are still unclear. Lifestyle-based interventions, including diet and aerobic and/or resistance training, have demonstrated to reduce development of type 2 diabetes in at-risk population, by improving glucose tolerance [19]. Moreover, some complementary studies have assessed interactions between physical activity and macronutrient distribution on fuel metabolism with impact on blood glucose $[16,17]$.

Personalized nutrition (PN) in obese and diabetic subjects aims to reduce body weight as well as mitigate hyperglycaemia, which may be affected not only by energy restriction but also by the inter-individual baseline differences in glucose levels and physical activity depending on genetics [20]. The different effects of carbohydrate consumption and physical activity on glycaemia are well known, although the joint influences of both factors on carbohydrate metabolism have been less investigated, in particular related to blood glucose regulatory genes. Thus, the aim of this study was to examine the interaction of macronutrient intake and physical activity, where also a genetic risk score (GRS) was computed, to describe effects on glucose metabolism.

\section{Materials and Methods}

The present research includes baseline data from the Food $4 \mathrm{Me}$ study [21] including participants from 7 European countries [22].

\section{Study Design}

Food4Me is the first large multi-centre RCT concerning a webbased PN approach [21]. The Food4me trial was conducted as a multi-centre, proof-of-principle study of PN to determine whether providing more personalized dietary advice leads to greater improvements in eating and health compared to conventional population-based advice. A total of 1,607 participants with a mean age of 39.8 years (ranging from 18 to 79 years) were enrolled. Of these participants, $60.9 \%$ were women and $96.7 \%$ were from white-European background. The mean BMI for all randomized participants was $25.5 \mathrm{~kg} / \mathrm{m}^{2}$, and $44.8 \%$ of the participants had a BMI $\geq 25.0 \mathrm{~kg} / \mathrm{m}^{2}$. The investigation collected auto-referenced anthropometrics data, self-declared dietary information, blood samples in dried cards, and oral cells collected using swabs. The GRS used in these analyses in this proof of principle was based only in 15 genes related with glucose utilization and not in all screened genotyped SNPs $(n=31)$. In brief, this investigation was designed as a prospective follow-up study with 4 intervention levels based on information provided to volunteers and 2 intensity levels depending on the frequency of advice [21]. The 4 levels of advice were level 0 , only general feedback and not based on the information provided by the volunteers; level 1 , feedback based on anthropometric and dietary intake data; level 2, level 1 and also biochemical information; and level 3, level 2 information and provided feedback based on the genetic makeup.
Navas-Carretero et al. 


\section{Participants}

Inclusion criteria were to be older than 18 years old, and exclusion criteria were minimum, to obtain a cohort representative of the adult population. Thus, the minimum exclusion criteria applied were pregnant or lactating; no or limited access to the Internet; following a prescribed diet for any reason, including weight loss, in the last 3 months; and diabetes, coeliac disease, Crohn's disease, or any metabolic disease or condition altering nutritional requirements such as thyroid disorders (if condition was not controlled), allergies, or food intolerances.

Of the total of 5,562 participants that registered online in the Food4Me study, only 2,764 completed the screening process and signed the 2 informed consent forms, and of those, 1,157 were excluded because they exceeded the sample size calculated (in chronological order at each intervention site, respecting the male/female ratio). From the 1,607 participants who were enrolled in the trial [21], 1,271 completed the 6-month intervention [23]. Out of the 336 dropouts, 127 were immediately after randomizing (10 of them due to unrealistic measures), 165 before the 3 months, and 46 before the end of the study. Only volunteers who finished the nutritional intervention and provided complete data on anthropometric measures and supplied adequate samples for biochemical and genetic analyses at baseline $(n=1,271)$ were included in the current analyses (Fig. 1).

\section{Dietary, Lifestyle, and Anthropometric Measures}

A validated Food Frequency Questionnaire (FFQ) and dietary habits questionnaire [24-26] were completed by participants to self-report dietary intake and habits, which focused on the present carbohydrate intake, as well as energy consumption. The FFQ was based on the EPIC-FFQ, and validated information related to macronutrients was used [24, 25].

Self-reported anthropometrical measures (weight, height, and waist circumference) were collected following validated procedures [27]. Moreover, participants completed the online Baecke questionnaire providing information about work, sports, and nonsport leisure activities during the last month to estimate the physical activity score ranging from 3 to 15 points [28, 29].

\section{Biochemical Parameters}

Fingertip fasting blood samples were self-collected on filter papers and dried (dried blood spots ) and afterwards posted to the recruitment centre and shipped to the analytical laboratories of Vitas and DSM (Vitas Ltd, Oslo, Norway; DSM NV, Heerlen, The Netherlands) for the determination of glucose, total cholesterol, carotenoids, and fatty acid patterns [30, 31].

\section{Genetic Characteristics}

Buccal cell samples were collected using swabs at baseline by all participants. LGC Genomics, Teddington, United Kingdom, performed DNA isolation and analyses of the samples by KASPTM genotyping assays following validated procedures, where 31 SNPs were screened.

\section{Ethics}

All the participating centres obtained ethical approval for the study protocol from their corresponding local research ethics committees. The Food4Me project was registered on February 9, 2012, with the Trial Registration: NCT01530139 at clinicaltrials. gov (http://clinicaltrials.gov/show/NCT01530139) and followed

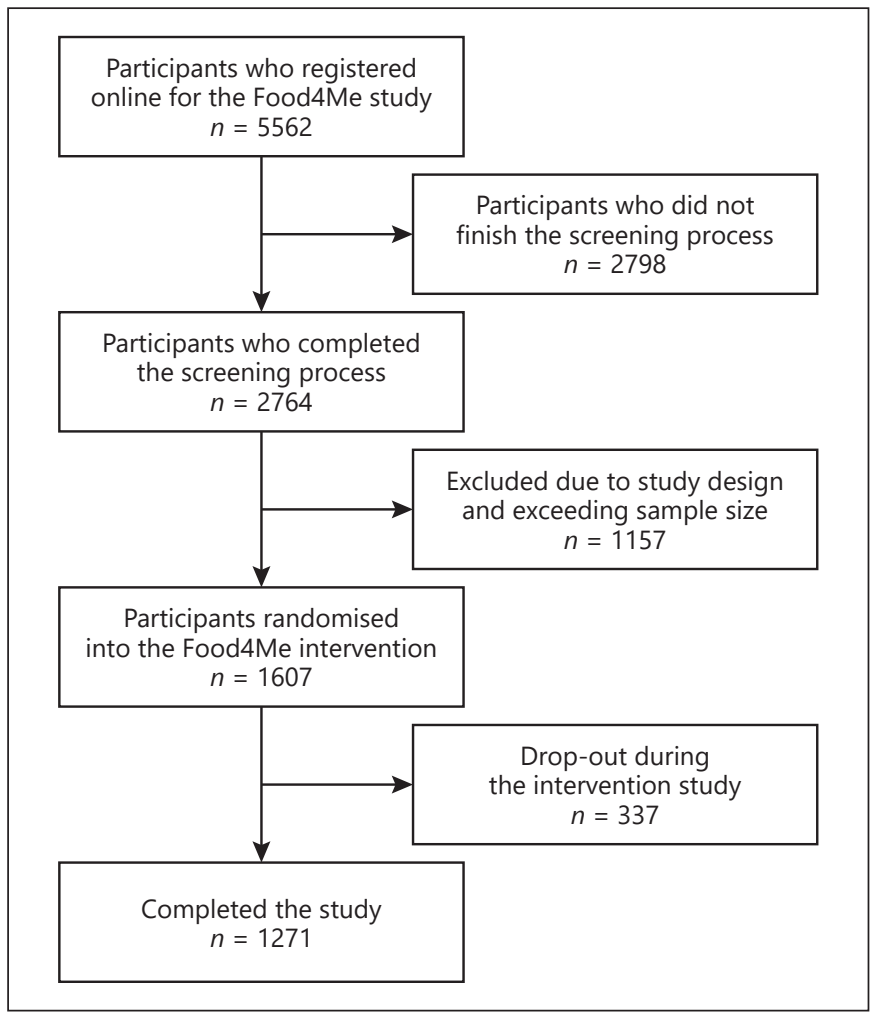

Fig. 1. Flowchart of the participants in the Food4Me study.

the CONSORT guidelines. The signature on 2 online consent forms was required for all the candidates interested in participating in the Food4Me study.

\section{Genetic Risk Score}

A GRS was "a priori" computed by adding the number of risk alleles concerning selected SNPs, which were initially chosen as related to glucose utilization traits. Such selection criteria meant that from 31 genes within the panel in Food4me (online suppl. Table 1; see www.karger.com/doi/10.1159/000515068 for all online suppl. material), only 15 meet the standards to be accounted on the devised GRS as related to glycaemia regulation, whose selection criteria are provided (online suppl. Table 2). Thus, the inclusion of SNPs for this proof-of-principle approach was based on attesting accepted statistical screening criteria based on associations $(p<0.10)$ of the carried risk allele numbers with the main variable (glycaemia), to be carried by at least $n=10$ subjects in each genotype, to appear in the OMIM database as involved in glucose metabolism, or to have identified at least 2 independent studies showing relationships with the investigated traits (online suppl. Table 2). All of them were found in HWE except for MAOA rs6323, although in this case, there is evidence of it being in equilibrium in European ancestries [32] or Caucasian [33] populations. The GRS analyses implemented as unweighted account for this conceptual research, given the lack of reliable and harmonized beta values for all SNPs. In any case, the analyses revealed a role for genetics in blood glucose determinations. 
Table 1. Characteristics of the participants in the Food4Me study according to demographic and anthropometric parameters $(n=1,271)$



Values are expressed as mean \pm SD or percentages. GRS, genetic risk score. ${ }^{*} p<0.05, t$ test comparisons of higher versus lower values (median of the values applied).

The SNPs included in generating the current GRS were the following (online suppl. Table 2): FTO (rs9939609 and rs1121980), FADS1 (rs174546), ApoE (rs429358), MTHFR (rs1801133), GPX1 (rs1050450), GSTP1 (rs1695), SLC6A4 (rs16965628), VDR (rs2228570), GC (rs2282679, rs4588, and rs7041), CETP (rs3764261), MAOA (rs6323), and BCMO1 (rs6564851). All the SNPs were selected because they were recognized to be involved in carbohydrate/energy metabolism (online suppl. Table 2), as found in gene cards, SNPedia, or PubMed [34], or showed compatible trends to significance in this population. The cutoff points were selected for both sexes, as well as by the median distribution in our sample, concerning factors later analysed (age, BMI, GRS, carbohydrate intake, and energy intake).

\section{Statistics}

Conventional descriptive statistics were carried out for comparisons of sex, age, and BMI, as well as for energy and carbohydrate intake, lifestyle (physical activity) criteria, and the GRS as relevant variables in this study. The sample characteristics concerning the distribution by sex, age, and BMI were tabulated and statistically analysed as well as the features concerning energy (kcal/day), carbohydrate (\% E), physical activity (Baecke scores units), and GRS (alleles account) as putative variables required for the regression analyses. Linear regression mixed analyses were performed at baseline to investigate the association between blood glucose levels and the GRS. Regression analyses were performed to discern the potential influence of different factors, such as age and sex (model 1), physical activity (model 2), carbohydrate intake (model 3), the GRS (model 4), and the interaction of physical ac- tivity and carbohydrate intake (model 5). A statistical interaction term (carbohydrate intake $\times$ physical activity level) was added to the model to investigate the role of the GRS, being the dependent variable of glucose levels, and independent variables carbohydrate intake and physical activity; we also adjusted for confounders such as age, sex, and BMI. Statistical analyses were performed using STATA statistical software (Stata IC version 12.0; StataCorp, College Station, TX, USA), and $p$ values $<0.05$ were considered significant.

\section{Results}

The characteristics of the study sample, total and dichotomized by sex or between high or low values of age, BMI, physical activity levels, GRS, and energy and carbohydrate intake, are reported (Tables 1, 2). Differences between groups related to age and BMI were observed, as well as anthropometric, biochemical, diet, lifestyle, and GRS. As hypothesized, there was a negative relationship between physical activity and blood glucose concentration, whereas there were positive correlations between blood glucose concentration and BMI as well as the GRS (Fig. 2) but not with carbohydrate intake alone. 


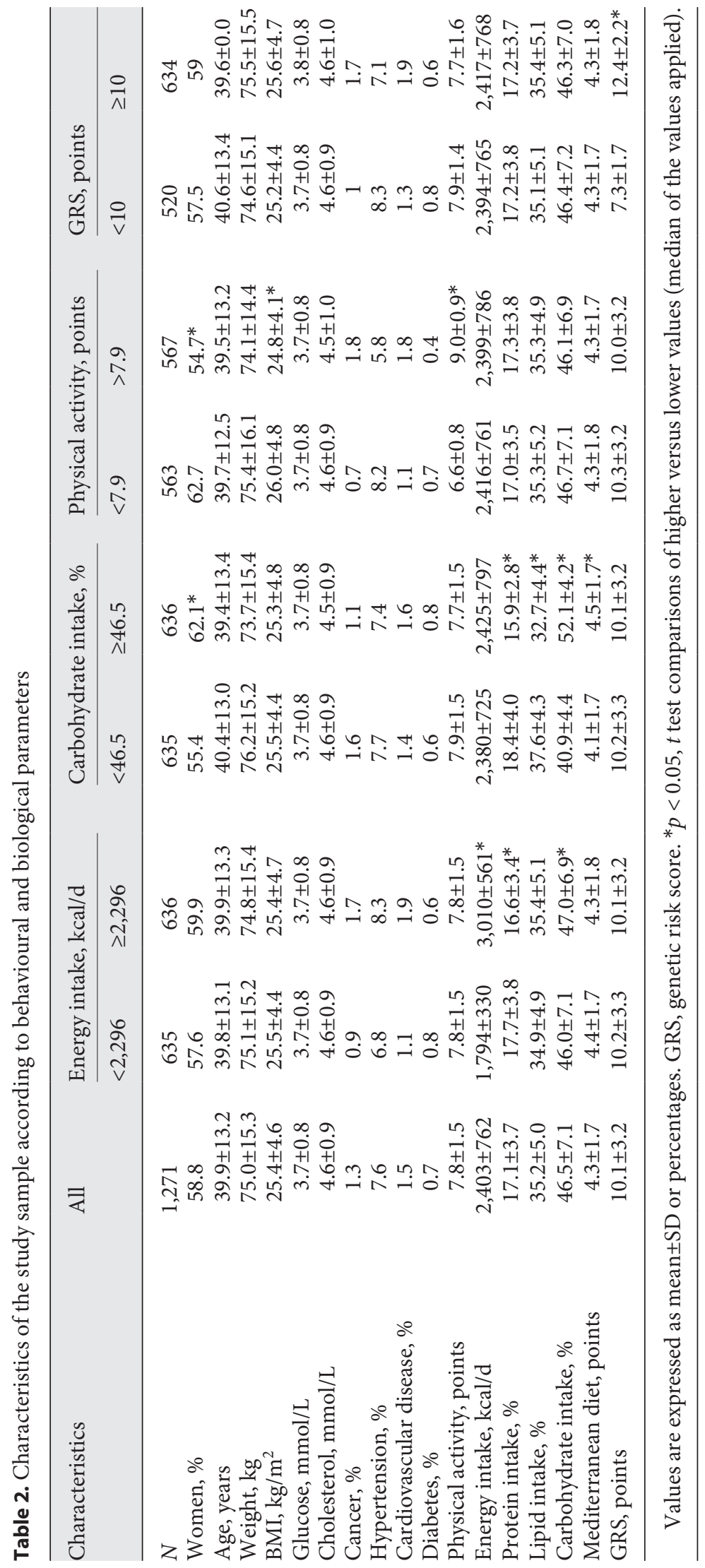






Fig. 2. Relationship between blood glucose concentrations and physical activity, carbohydrate intake, BMI, and the GRS. Statistical analyses were performed to investigate the associations at baseline between blood glucose levels with carbohydrate intake (\% E), BMI $\left(\mathrm{kg} / \mathrm{m}^{2}\right)$, and GRS (sum of risk alleles). GRS, genetic risk score.

Fig. 3. Interaction of blood glucose concentrations and physical activity level depending on the median carbohydrate intake ( $p$ for interaction $=0.0033$ ), where the median $\mathrm{CHO}$ intake was $46.5 \% \mathrm{E}$.

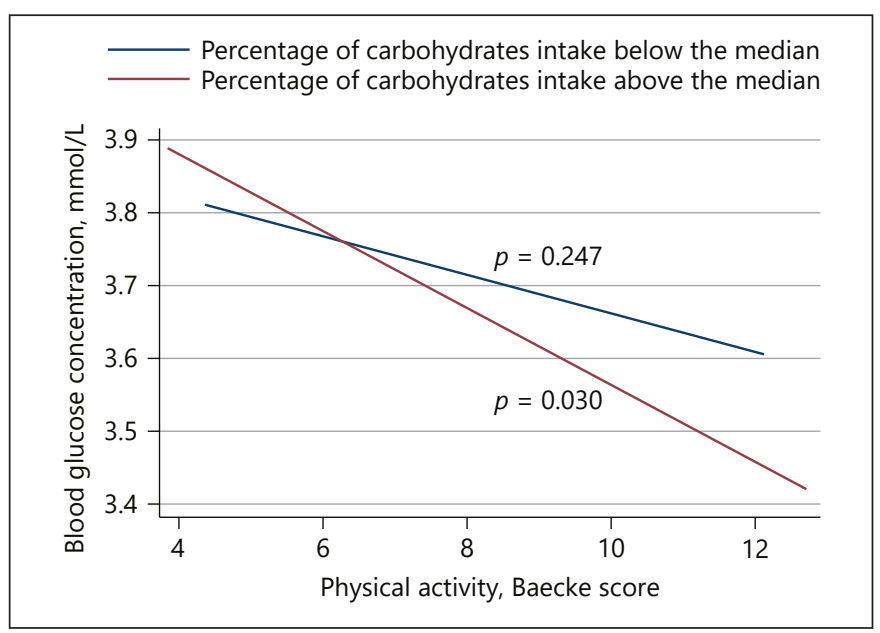


Regarding the regression analyses (Table 3), the contribution of genetic, dietary, and lifestyle factors was quantified in the model 5 to being significant contributors, the GRS $(1.5 \%, p=0.047)$ and the interaction between physical activity and carbohydrate intake $(-6.0 \%$, $p=0.033)$ to blood glucose concentration. The interaction between physical activity and carbohydrate consumption is illustrated in Figure 3 and should be interpreted as the most relevant result in the current analyses.

\section{Discussion}

Our data indicate an interaction between physical activity and carbohydrate intake on circulating blood glucose concentration, which is influenced by individual genetic makeup as expressed in the GRS. These results suggest that glycaemia may depend on interplay of both modifiable factors as the product term (carbohydrate consumption $\times$ energy expenditure) is associated with the degree of physical activity.

$\mathrm{PN}$ involves integration of phenotypical and genotypical data as well as individual food preferences and the clinical background, to provide precise nutritional management of healthy and diseased subjects [35]. Thus, identifying traits such as glycaemia with interpersonal differences affecting energy metabolism may result in a more individualized management of obesity and diabetic complications [35], depending on the macronutrient distribution as well as on the genotype [36].

The Food4Me project was designed to evaluate the role of individualized approaches using internet tools [37]. This strategy displayed some interesting benefits on providing nutritional advice, also related to genetics [31], anthropometric determinations [38], biochemical parameters like glucose and cholesterol $[39,40]$, and lifestyle information concerning physical activity and dietary intake $[39,41,42]$. A GRS was built with 15 recognized genes involved in $\mathrm{CHO}$ metabolism or energy-related processes with a potential impact on glucose metabolism [43].

In research on genetic determinants concerning insulin [43], some metabolic features like hyperglycaemia have been screened through linkage studies, candidate genes association studies, GWAS, exome sequencing, and epigenetic approaches [44], as well as by meta-analyses in Asian and European populations [45], where the genetic makeup exhibited a role in glycaemic control. Physical activity may exert beneficial effects on glucose metabolism via specific genes, which may impact glucose levels and risk of developing full-blown diabetes [46] or cardiometabolic conditions [47].

Intake, Physical Activity, and Genetics in Relation to Glucose Metabolism
Table 3. Linear regression analyses between blood glucose concentrations and genetic, dietary, and lifestyle factors

\begin{tabular}{|c|c|c|}
\hline Model & Coefficient $\beta$ & $p$ value \\
\hline \multicolumn{3}{|l|}{ Model 1} \\
\hline Age & 0.008 & $<0.001$ \\
\hline Sex & -0.029 & 0.560 \\
\hline \multicolumn{3}{|l|}{ Model 2} \\
\hline Age & 0.007 & 0.001 \\
\hline Sex & -0.049 & 0.329 \\
\hline Physical activity & -0.041 & 0.013 \\
\hline \multicolumn{3}{|l|}{ Model 3} \\
\hline Age & 0.007 & 0.001 \\
\hline Sex & -0.047 & 0.353 \\
\hline Physical activity & -0.041 & 0.013 \\
\hline Carbohydrate intake & -0.186 & 0.595 \\
\hline \multicolumn{3}{|l|}{ Model 4} \\
\hline Age & 0.006 & 0.001 \\
\hline Sex & -0.057 & 0.260 \\
\hline Physical activity & -0.037 & 0.025 \\
\hline Carbohydrate intake & -0.193 & 0.583 \\
\hline GRS & 0.015 & 0.050 \\
\hline \multicolumn{3}{|l|}{ Model 5} \\
\hline Age & 0.006 & 0.001 \\
\hline Sex & -0.051 & 0.312 \\
\hline GRS & 0.015 & 0.047 \\
\hline $\begin{array}{l}\text { Interaction physical activity- } \\
\text { carbohydrate intake }\end{array}$ & -0.060 & 0.033 \\
\hline
\end{tabular}

A regression analysis was performed to examine the potential influence of different factors, such as age and sex (model 1), physical activity (model 2), carbohydrate intake (model 3), GRS (model 4), and the interaction of physical activity and carbohydrate intake (model 5), on glycaemia. A statistical interaction term (carbohydrate intake $\times$ physical activity level) was added to the model to investigate the role of the GRS, being the dependent variable of glucose levels, and independent variables carbohydrate intake and physical activity, as well as adjusting for confounders such as age, sex, and reported BMI. GRS, genetic risk score.

High intake of carbohydrates and glycaemia have been related to enhanced glucose oxidation and reduced lipid utilization, where different mechanisms have been involved such as muscle glucose uptake and phosphorylation-mediated gluconeogenesis, glycolysis, glycogen storage, and conversion to acetyl CoA depending on exercise [48]. The shared pathways of carbohydrate and lipid metabolism during exercise are less well known, where transport of fat into the muscle, specific binding proteins for mitochondrial translocation of fat, and new roles for lipases have been investigated [49].

Interactions between physical activity and macronutrient composition of energy-restricted diets may affect body fat reduction and blood glucose concentration be- 
cause less-active individuals with a high-carbohydrate/ low-fat diet showed greater fat loss than those more active with a lower-CHO/high-fat diet [17]. These findings support the notion that precision nutrition for control of glycaemia or body weight may benefit from considering phenotypic as well as genotypic data $[20,50]$.

Gene-environment interactions in energy and glucose homeostasis are relevant for understanding glucose/fat utilization during exercise [47], with important influence on health outcomes [51]. Moreover, the interplay between degree of physical activity and dietary fat intake could play a role to understand the influence of dietary carbohydrates on energy balance [52], but in our case, the effects were not statistically significant (data not shown). One of the most novel results of the current analyses is that in addition to the influence of physical activity and the genetic background on blood glucose concentration, we observed an interaction between physical activity and the proportion of energy supplied by carbohydrates.

Our study has some limitations like the online questionnaire completion, although the procedure was validated $[25,27]$. Moreover, we only used 15 SNPs from the panel, although scientific, statistical, genetic, and epidemiological cautions and criteria implemented as previously detailed to produce this proof of concept revealed that genes regulate energy utilization depending on carbohydrate intake and physical activity. Furthermore, the study could benefit from separate analyses of total carbohydrates and simple sugars, which was not achievable by the features of the food composition database, neither measurements of the body fat content.

The Food4Me study also exhibits some strengths like a fairly high number of individuals included from 7 different European communities [21]. In conclusion, blood glucose concentration is under influence of some specific genes related to carbohydrate metabolism, as included in the GRS, physical activity, and the proportion of carbohydrate intake.

\section{Acknowledgment}

Sharron Kuznesof, Arnout R.H. Fischer, Aleksandra Berezowska, Ivo vander Lans, Maria Daniel Vaz de Almeida, Bruno Oliveira, Rui Poínhos, Marian Raley, Maresa Duffy, Karen Orr, and Jenny Robinson are gratefully acknowledged for developing the questionnaires included in the Food4Me Study.

\section{Statement of Ethics}

All procedures performed in the study were in accordance with the ethical standards of the corresponding research committees of each of the 7 participating centres and with the 1964 Helsinki Dec- laration and its later amendments or comparable ethical standards. Informed consent was obtained from all individual participants included in the study. The Research Ethics Committees evaluating the study protocol were those with the appropriate authority in each study site: University College Dublin, Ireland (Ref No. LS12-05-Gibney-Gibney); University of Maastricht, Netherlands (Ref No. NL39135.068.12); Universidad de Navarra, Spain (Ref No. 041/2012); Harokopio University, Greece (Ref No. 34/02-042012); The University of Reading, United Kingdom (Ref No. 12/09); National Food and Nutrition Institute, Poland (Ref No. 61/63); and Technische Universitaet Muenchen, Germany (Ref No. 5332/11). With the study coordinator being in Ireland, the relevant Health Authority in the Food4Me study was the Research Ethics Committee of Ireland.

\section{Conflict of Interest Statement}

C.A.D. is a cofounder, board member, stockowner, and consultant for Vitas Ltd. (http://vitas.no) performing the dried blood spot analyses. T.E.G. is a cofounder, stockowner, and CEO of Vitas.

\section{Funding Sources}

The Food4Me project has received funding from the European Union's Seventh Framework Programme for research, technological development, and demonstration (Grant Agreement No. 265494). The research leading to these results has received funding from "la Caixa" Banking Foundation through a grant for the $\mathrm{PhD}$ work of R.S.-C.

\section{Author Contributions}

R.S.-C., I.A.-A., and S.N.-C. drafted the manuscript and performed the statistical analysis for the manuscript. J.A.M. was responsible of the Spanish centre of intervention. M.G., J.C.M., J.A.M., S.N.-C., C.C.M., M.C.W., E.R.G., L.B., W.H.M.S., H.D., L.J.F., B.S.K., C.A.D., J.A.L., Y.M., and M.J. contributed to the research design. R.S.-C., S.N.-C., K.M.L., A.R., C.F.M.M., C.O'.D., H.F., C.W., A.L.M., R.F., G.M., and C.P.L. conducted the intervention. T.E.G. performed most of the biochemical measurements. All authors contributed to a critical review of the manuscript during the writing process. All authors approved the final version to be published.

\section{Availability of Data and Material}

Requests for data and material should be directed to the study director Professor Mike Gibney (mike.gibney@ucd.ie) and/or Professor John Mathers (john.mathers@newcastle.ac.uk). 


\section{References}

1 Hall KD, Guo J. Obesity energetics: body weight regulation and the effects of diet composition. Gastroenterology. 2017;152:171827.e3.

2 Misra A, Soares MJ, Mohan V, Anoop S, Abhishek V, Vaidya R, et al. Body fat, metabolic syndrome and hyperglycemia in South Asians. J Diabetes Complications. 2018;32: 1068-75.

3 González-Muniesa P, Mártinez-González MA, Hu FB, Després JP, Matsuzawa Y, Loos RJF, et al. Obesity. Nat Rev Dis Primers. 2017; 3:17034.

4 Pan B, Ge L, Xun YQ, Chen YJ, Gao CY, Han $\mathrm{X}$, et al. Exercise training modalities in patients with type 2 diabetes mellitus: a systematic review and network meta-analysis. Int J Behav Nutr Phys Act. 2018;15:72.

5 Martinez JA, Navas-Carretero S, Saris WH, Astrup A. Personalized weight loss strategiesthe role of macronutrient distribution. Nat Rev Endocrinol. 2014;10:749.

6 Gardner CD, Trepanowski JF, Del Gobbo LC, Hauser ME, Rigdon J, Ioannidis JPA, et al. Effect of low-fat vs low-carbohydrate diet on 12 -month weight loss in overweight adults and the association with genotype pattern or insulin secretion: the DIETFITS randomized clinical trial. JAMA. 2018;319:667-79.

7 Saris WH, Astrup A, Prentice AM, Zunft HJ, Formiguera X, Verboeket-van de Venne WP, et al. Randomized controlled trial of changes in dietary carbohydrate/fat ratio and simple vs complex carbohydrates on body weight and blood lipids: the CARMEN study. The Carbohydrate Ratio Management in European National diets. Int J Obes Relat Metab Disord. 2000;24:1310-8.

8 Larsen TM, Dalskov SM, van Baak M, Jebb SA, Papadaki A, Pfeiffer AF, et al. Diets with high or low protein content and glycemic index for weight-loss maintenance. N Engl J Med. 2010;363:2102-13

9 Thorsdottir I, Tomasson H, Gunnarsdottir I, Gisladottir E, Kiely M, Parra MD, et al. Randomized trial of weight-loss-diets for young adults varying in fish and fish oil content. Int J Obes. 2007;31:1560-6.

10 Feldman AL, Long GH, Johansson I, Weinehall L, Fhärm E, Wennberg P, et al. Change in lifestyle behaviors and diabetes risk: evidence from a population-based cohort study with 10 year follow-up. Int J Behav Nutr Phys Act. 2017;14:39.

11 de Luis DA, Izaola O, Primo D, Aller R. Different effects of high-protein/low-carbohydrate versus standard hypocaloric diet on insulin resistance and lipid profile: Role of rs16147 variant of neuropeptide Y. Diabetes Res Clin Pract. 2019;156:107825.

12 Shungin D, Winkler TW, Croteau-Chonka DC, Ferreira T, Locke AE, Mägi R, et al. New genetic loci link adipose and insulin biology to body fat distribution. Nature. 2015;518: 187-96.
13 Webb VL, Wadden TA. Intensive lifestyle intervention for obesity: principles, practices, and results. Gastroenterology. 2017;152: 1752-64.

14 Qi L, Hu FB, Hu G. Genes, environment, and interactions in prevention of type 2 diabetes: a focus on physical activity and lifestyle changes. Curr Mol Med. 2008;8:519-32.

15 Tan PY, Mitra SR, Amini F. Lifestyle interventions for weight control modified by genetic variation: a review of the evidence. Public Health Genomics. 2018;21:169-85.

16 Abdel-Hamid TK. Exercise and diet in obesity treatment: an integrative system dynamics perspective. Med Sci Sports Exerc. 2003; 35:400-13.

17 López-Fontana CM, Sánchez-Villegas A, Martínez-Gonzalez MA, Martinez JA. Daily physical activity and macronutrient distribution of low-calorie diets jointly affect body fat reduction in obese women. Appl Physiol Nutr Metab. 2009;34:595-602.

18 Jacobs DR, Sluik D, Rokling-Andersen $\mathrm{MH}$, Anderssen SA, Drevon CA. Association of 1-y changes in diet pattern with cardiovascular disease risk factors and adipokines: results from the 1-y randomized oslo diet and exercise study. Am J Clin Nutr. 2009;89:509-17.

19 Aguiar EJ, Morgan PJ, Collins CE, Plotnikoff RC, Callister R. Efficacy of interventions that include diet, aerobic and resistance training components for type 2 diabetes prevention: a systematic review with meta-analysis. Int J Behav Nutr Phys Act. 2014;11:2.

20 Zeevi D, Korem T, Zmora N, Israeli D, Rothschild D, Weinberger A, et al. Personalized nutrition by prediction of glycemic responses. Cell. 2015;163:1079-94.

21 Celis-Morales C, Livingstone KM, Marsaux CF, Forster H, O’Donovan CB, Woolhead C, et al. Design and baseline characteristics of the Food4Me study: a web-based randomised controlled trial of personalised nutrition in seven European countries. Genes Nutr. 2015; $10: 450$.

22 Celis-Morales C, Livingstone KM, Marsaux CF, Macready AL, Fallaize R, O'Donovan CB, et al. Effect of personalized nutrition on health-related behaviour change: evidence from the Food4Me European randomized controlled trial. Int J Epidemiol. 2017;46:57888.

23 Livingstone KM, Celis-Morales C, MacReady AL, Fallaize R, Forster H, Woolhead C, et al. Characteristics of European adults who dropped out from the Food4Me Internetbased personalised nutrition intervention. Public Health Nutr. 2017;20:53.

24 Forster H, Walsh MC, O'Donovan CB, Woolhead C, McGirr C, Daly EJ, et al. A dietary feedback system for the delivery of consistent personalized dietary advice in the web-based multicenter Food4Me study. J Med Internet Res. 2016;18.
25 Marshall SJ, Livingstone KM, Celis-Morales C, Forster H, Fallaize R, O'Donovan CB, et al. Reproducibility of the online Food4Me foodfrequency questionnaire for estimating dietary intakes across Europe. J Nutr. 2016;146: 1068.

26 Fallaize R, Forster H, Macready AL, Walsh MC, Mathers JC, Brennan L, et al. Online dietary intake estimation: reproducibility and validity of the Food4Me food frequency questionnaire against a 4-day weighed food record. J Med Internet Res. 2014;16(8):e190.

27 Celis-Morales C, Livingstone KM, Woolhead C, Forster H, O'Donovan CB, Macready AL, et al. How reliable is internet-based self-reported identity, socio-demographic and obesity measures in European adults? Genes Nutr. 2015;10:28.

28 Marsaux CF, Celis-Morales C, Hoonhout J, Claassen A, Goris A, Forster H, et al. Objectively measured physical activity in European adults: Cross-sectional findings from the food4me study. PLoS One. 2016;11:e0150902.

29 Marsaux CF, Celis-Morales C, Livingstone KM, Fallaize R, Kolossa S, Hallmann J, et al. Changes in physical activity following a genetic-based internet-delivered personalized intervention: randomized controlled trial (Food4Me). J Med Internet Res. 2016;18:e30.

30 Markussen MS, Veierød MB, Sakhi AK, Ellingjord-Dale $\mathrm{M}$, Blomhoff $\mathrm{R}$, Ursin $\mathrm{G}$, et al. Evaluation of dietary patterns among Norwegian postmenopausal women using plasma carotenoids as biomarkers. Br J Nutr. 2015; 113:672-82.

31 Albani V, Celis-Morales C, Marsaux CF, Forster H, O'Donovan CB, Woolhead C, et al. Exploring the association of dairy product intake with the fatty acids C15:0 and C17:0 measured from dried blood spots in a multipopulation cohort: findings from the Food4Me study. Mol Nutr Food Res. 2016;60: 834.

32 Tomasi J, Zai CC, Zai G, Herbert D, King N, Freeman N, et al. The effect of polymorphisms in startle-related genes on anxiety symptom severity. J Psychiatr Res. 2020;125: $144-51$.

33 Balestri M, Calati R, Serretti A, Hartmann AM, Konte B, Friedl M, et al. Maoa and Maob polymorphisms and personality traits in suicide attempters and healthy controls: a preliminary study. Psychiatry Res. 2017;249: 212-7.

34 Grimaldi KA, van Ommen B, Ordovas JM, Parnell LD, Mathers JC, Bendik I, et al. Proposed guidelines to evaluate scientific validity and evidence for genotype-based dietary advice. Genes Nutr. 2017;12:35.

35 de Toro-Martín J, Arsenault BJ, Després JP, Vohl MC. Precision nutrition: a review of personalized nutritional approaches for the prevention and management of metabolic syndrome. Nutrients. 2017;9:913. 
36 Martinez JA, Navas-Carretero S, Saris WH, Astrup A. Personalized weight loss strategiesthe role of macronutrient distribution. Nat Rev Endocrinol. 2014;10:749-60.

37 Celis-Morales C, Livingstone KM, Marsaux CF, Macready AL, Fallaize R, O’Donovan CB, et al. Effect of personalized nutrition on health-related behaviour change: evidence from the Food4Me European randomized controlled trial. Int J Epidemiol. 2017;46:578.

38 Livingstone KM, Celis-Morales C, NavasCarretero S, San-Cristobal R, Forster $\mathrm{H}$, O'Donovan CB, et al. Fat mass- and obesityassociated genotype, dietary intakes and anthropometric measures in European adults: the Food4Me study. Br J Nutr. 2015;115:440.

39 Fallaize R, Livingstone KM, Celis-Morales C, Macready AL, San-Cristobal R, Navas-Carretero S, et al. Association between diet-quality scores, adiposity, total cholesterol and markers of nutritional status in european adults: findings from the Food4Me study. $\mathrm{Nu}$ trients. 2018;10(1).

40 Albani V, Celis-Morales C, O’Donovan CB, Walsh MC, Woolhead C, Forster $\mathrm{H}$, et al. Within-person reproducibility and sensitivity to dietary change of C15:0 and C17:0 levels in dried blood spots: data from the European Food4Me Study. Mol Nutr Food Res. 2017;61.
41 Celis-Morales C, Marsaux CF, Livingstone KM, Navas-Carretero S, San-Cristobal R, Fallaize $\mathrm{R}$, et al. Can genetic-based advice help you lose weight? Findings from the Food4Me European randomized controlled trial. Am J Clin Nutr. 2017;105:1204.

42 Kirwan L, Walsh MC, Celis-Morales C, Marsaux CF, Livingstone KM, Navas-Carretero S, et al. Phenotypic factors influencing the variation in response of circulating cholesterol level to personalised dietary advice in the Food4Me study. Br J Nutr. 2016;116:2011.

43 Shungin D, Winkler TW, Croteau-Chonka DC, Ferreira T, Locke AE, Mägi R, et al. New genetic loci link adipose and insulin biology to body fat distribution. Nature. 2015;518: 187-96.

44 Fathi Dizaji B. The investigations of genetic determinants of the metabolic syndrome. Diabetes Metab Syndr. 2018;12:783-9.

45 Hong KW, Chung M, Cho SB. Meta-analysis of genome-wide association study of homeostasis model assessment $\beta$ cell function and insulin resistance in an East Asian population and the European results. Mol Genet Genomics. 2014;289:1247-55.
46 Kilpeläinen TO, Franks PW. Gene-physical activity interactions and their impact on diabetes. Med Sport Sci. 2014;60:94-103.

47 Sarzynski MA, Loos RJ, Lucia A, Pérusse L, Roth SM, Wolfarth B, et al. Advances in exercise, fitness, and performance genomics in 2015. Med Sci Sports Exerc. 2016;48:1906-16.

48 Spriet LL, Watt MJ. Regulatory mechanisms in the interaction between carbohydrate and lipid oxidation during exercise. Acta Physiol Scand. 2003;178:443-52.

49 Spriet LL. New insights into the interaction of carbohydrate and fat metabolism during exercise. Sports Med. 2014;44:S87-96.

50 de Toro-Martín J, Arsenault BJ, Després JP, Vohl MC. Precision nutrition: a review of personalized nutritional approaches for the prevention and management of metabolic syndrome. Nutrients. 2017;9:913.

51 Smith HA, Gonzalez JT, Thompson D, Betts JA. Dietary carbohydrates, components of energy balance, and associated health outcomes. Nutr Rev. 2017;75:783-97.

52 Astrup A. Macronutrient balances and obesity: the role of diet and physical activity. Public Health Nutr. 1999;2:341-7. 